\title{
Study of Subjective and Objective Quality Evaluation of 3D Point Cloud Data by the JPEG Committee
}

\author{
Stuart Perry; University of Technology Sydney; Sydney; Australia; António Pinheiro; Universidade da Beira Interior; Covilhã; \\ Portugal; Emil Dumic; University North; Varazdin; Croatia; Luis A. da Silva Cruz; Universidade de Coimbra; Coimbra; Portugal
}

\begin{abstract}
The SC29/WG1 (JPEG) Committee within ISO/IEC is currently working on developing standards for the storage, compression and transmission of $3 D$ point cloud information. To support the creation of these standards, the committee has created a database of $3 D$ point clouds representing various quality levels and use-cases and examined a range of $2 D$ and $3 D$ objective quality measures. The examined quality measures are correlated with subjective judgments for a number of compression levels. In this paper we describe the database created, tests performed and key observations on the problems of $3 D$ point cloud quality assessment.
\end{abstract}

\section{Introduction}

The ISO/IEC/SC29/WG1 (JPEG) Committee is tasked with advancing still image coding standards. This group was responsible for creating the JPEG image compression standard, the dominant compression format for still images today. Part of JPEG Committee's mandate is to further improve the JPEG standard and advance still image standards to new sources of image data such as plenoptic imagery, holography, 360 degree images and 3D point clouds. With the rise of Virtual, Augmented and Mixed Reality technology, the efficient storage and transmission of vast quantities of $3 \mathrm{D}$ point cloud data will become vital to fully realizing the new applications of this technology. Hence the JPEG Committee has created an Ad hoc Group (JPEG Pleno Point Cloud AhG) to study the efficient storage and transmission of 3D point cloud (PC) data. An important part of the mandate of this group is to study effective methods of measuring quality and fidelity of point clouds.

Given that point cloud image quality evaluation is not well studied, one of the early mandates of the JPEG Pleno Point Clouds Ad hoc Group (AhG) was to collect a suitable database of 3D point clouds and respective subjective quality scores and examine issues of quality assessment for point clouds.

Another important early mandate for the group was the identification of key use cases for point cloud coding and from these use-cases identify common requirements of future point cloud coding proposals. Key use-cases identified by the group include usecases in the areas of:

- Manufacturing - traditional and additive systems

- On-line shopping

- Fault and defect detection in manufacturing and construction

- Cultural Heritage

- Wide-area survey/3D mapping

From study of the above use-cases, the group has identified the following key requirements of any future static point cloud coding proposal:

- $\quad$ Support for coding and compression of both local and global attributes as well as geometric information
- Tunable Quality

- Scalability of Geometry and Attributes

- Different degrees of precision, resolution and range for both geometry and attributes

- Random Access - Selective decoding of a portion of the point cloud independently of the rest

In the next two sections we first describe the JPEG Pleno Point Clouds dataset and then provide a summary of the most common point cloud compression methods, some of which were used to prepare the distorted point clouds in the subjective experiment described later. The following section then describes some of the most common objective measures of point cloud quality, with emphasis on the ones used in the studies carried out so far by the JPEG Pleno Point Clouds AhG. The next to last section describes the point clouds subjective quality studies carried out so far with an analysis of their results. Finally, we close the article with a conclusion section.

\section{JPEG Pleno Point Cloud Database}

The JPEG Pleno Point Cloud AhG solicited and collected a database of point cloud data [1]. This includes both natural and artificial content and has been vetted by the JPEG committee to represent a useful breadth of content concerning a number of key use-cases for designing and evaluating point cloud coding proposals.

The database consists of more than 30 point clouds and contains both artificially generated point clouds and content scanned from real-life scenes and objects. Database contents have been contributed by $8 \mathrm{i}$ Labs, Microsoft, Universidad Politécnica de Madrid, ScanLAB Projects, and the University of São Paulo [1]. Figure 1 shows examples of point clouds in the JPEG Pleno Point Cloud Database.

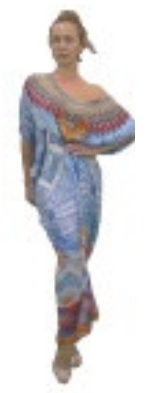

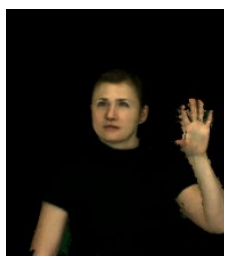

(b)

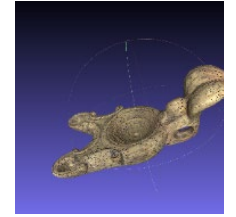

(c) (a) 


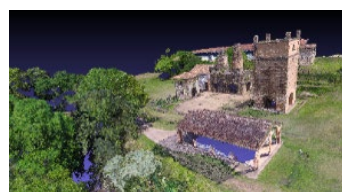

(d)

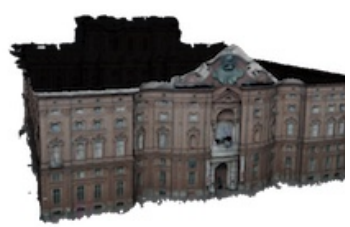

(e)

Figure 1: Examples of point clouds in the JPEG Pleno Point Cloud Database. (a) Example from the 8i Voxelized Full Bodies Dataset, (b) Example from the Microsoft Voxelized Upper Bodies Dataset, (c) Example from the University of São Paulo Point Cloud Dataset, (d) Example from the University of São Paulo Point Cloud Dataset and (e) Example from the GTI-UPM Point-cloud dataset.

\section{Background on Point Cloud Coding}

\section{Overview}

As a consequence of the large point count and the need to store position and attributes like color information for each point, typical raw point clouds require large amounts of storage, mandating the use of compression for efficient handling, transmission and storage.

The following paragraphs summarize the major point cloud compression methods reported in the literature. Compression methods can be specialized to compress only point position information (geometry only) or be able to compress both point position and attribute information. In an attempt to systematize the exposition, the surveyed methods were divided into three classes: methods based on spatial position encoding, methods based on projections and other methods.

\section{Methods based on 3D spatial/position encoding}

Methods in this class retain the spatial relationship between the points in the point cloud, e.g. by first determining the space region occupied by the $3 \mathrm{D}$ cloud of points which is then recursively partitioned into non-overlapping sub-regions up to a desired level of detail and encoding the partitioning structure using a binary code. The points in the smallest sub-regions can then be represented by some type of average point thus achieving lossy compression. Octree-based methods do exactly this by partitioning the point cloud bounding box into equal-sized eight smaller boxes and repeating the procedure when the occupancy status of each sub-box, or the desired geometrical accuracy of the representation justifies it. Another subset of methods in this class encode point neighborhood relationships, as in mesh-based compression algorithms where the point cloud is first converted into a mesh which is then compressed using mesh-specific compression procedures.

\section{Octrees}

Octree structures are very popular representations for point cloud data [2], [3] with support by point cloud libraries like PCL [4]. An octree represents a point cloud by partitioning the threedimensional space by recursively subdividing it into octants, according to a tree structure in which each internal node has eight children. Besides compression, octrees allow efficient processing of point clouds, such as fast search and cloud simplification by point pruning or resampling. It introduces compression if an octants' points are represented by a smaller set of the original or computed points. Octree structure encodings are inherently embedded, which enables scalable compression.

The algorithm in [2], encodes the geometry information of the tree nodes and leaves after each region subdivision. The local connectivity is encoded by a specific encoder.
Other methods like [5], [6], [7] and [8] use sub-partitioning structure prediction to encode the octree details. Works [5] and [6], first compute a local surface approximation and then apply prediction only to nearby regions. [7] follows a similar approach but the prediction is constrained to maximize tangent-plane continuity. Smith et al. propose in [8] the use of marching cubes to estimate the boundaries of each cell of the octree. Kammerl et al. [9] proposed to describe the volume occupied by the point cloud using octrees. Their work is targeted at dynamic point clouds and the temporal redundancy of consecutive point clouds octrees is exploited to increase the compression factor. In this method the color attributes are encoded using a simple entropy encoder. More recently Queiroz and Chou [10] proposed encoding the geometry of the point cloud using octree scanning and compressing the color attributes with a hierarchical transform and arithmetic encoder. In many methods prior to the octree decomposition the point cloud undergoes a voxelization step [5] [6], akin to a spatial quantization, which reduces the accuracy of the points position information.

\section{Meshes}

Point clouds can be compressed by first converting them to polygonal meshes and then compressing the meshes using one of several methodologies. Most meshes use triangles as the polygons of choice, which represent the point connectivity information. Attributes can be stored together with mesh vertices or mesh polygons. However, converting a point cloud to a polygonal mesh is a computationally complex process that impedes or limits realtime applications. Mesh compression can be achieved by reducing the number of points through the use of mesh simplification methods which do not change the local connectivity. Surveys of such methods can be found in [11] [12] [13]. This kind of mesh reduction method inevitably changes the positions of the original points, causing distortion. In [14], Rusinkiewicz and Levoy proposed the QSplat method, which starts with a triangular mesh representing the point cloud from which a bounding spheres structure is derived. The bounding spheres structure and node attributes are then encoded. In [15] Rossignac proposes the Edgebreaker method to compress the connectivity information of triangle meshes. This method requires no more than 2 bits per mesh triangle. In some variants Edgebreaker uses vertex data compression schemes to further reduce the data volume.

\section{Graphs}

Graph based signal transforms can also be used in point cloud compression with or without prior conversion to meshes. In [16], Karni and Gotsman describe graph-based methods for mesh geometric information compression. Recently [17] used a graph transform to compress the attribute information of a point cloud with the geometry encoded using octrees. Cohen et al. [18] voxelize the point clouds and then apply octrees to obtain a sparse set of blocks which is encoded using a graph transform. The attributes of the points in each block are encoded using a shape adaptive transform coupled with a graph transform. More recently [19] represents a dynamic point cloud data sequence as a set of graphs and encode point position and attributes as signals defined on the graph's vertices. Queiroz et al. [20] use a Gaussian Process Transform as the basis of graph transform coding of point clouds. A latter work [21] by Rente et al. proposed a layered method for encoding points clouds decomposed into octrees, where a base layer provides a lower quality representation of the point cloud and a second layer encoded using a graph transform adds quality enhancement information. 


\section{Methods based on projections}

Several methods for projection-based point cloud coding have been proposed recently. In [22] authors propose a best effort projection-based compression method for point clouds. To take advantage of the well-developed 2D compression algorithms, a regularized $3 \mathrm{D}$ point cloud is projected onto specified planes as different views while position information and related attributes are preserved. Joint depth- and color-dependent block-wise prediction was also utilized to further reduce the inter-view redundancy between projected 2D images. Point clouds are then successfully reconstructed via a corresponding decoding process. In paper [23] the authors present novel point cloud reduction methods based on panorama images generated using either equirectangular projection, cylindrical projection, Mercator projection, rectilinear projection, Pannini projection, stereographic projection or Albers equal-area conic projection. Different compression ratios can be achieved by using different resolutions for the panorama images. It is shown that the reduced point clouds are ideally suited for feature-based registration on panorama images. In [24] the same authors propose the use of conventional image-based compression methods for 3D point clouds. The point cloud is mapped onto panorama images using equirectangular projection, to encode the range, reflectance and color value for each point. Results of several lossless compression methods and lossy JPEG on point cloud compression were presented. Lossless compression methods are designed to retain the original data, while lossy compression methods sacrifice the details for a higher compression ratio. Figure 2 shows several representations of the "Longdress_1300" pointcloud [25] after compression using the projection-based 3DTK-toolkit [26] compressor, using the scan_to_panorama and panorama_to_scan tools. Figure 3 shows panorama images for equirectangular and azimuthal projection types.

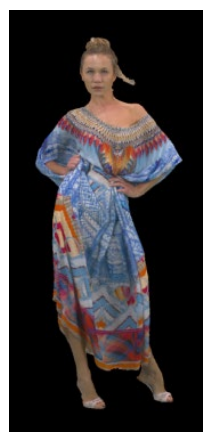

(a)

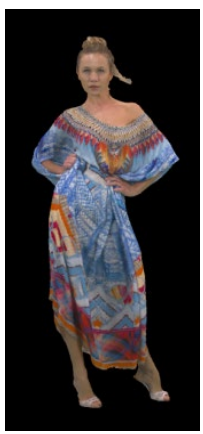

(b)

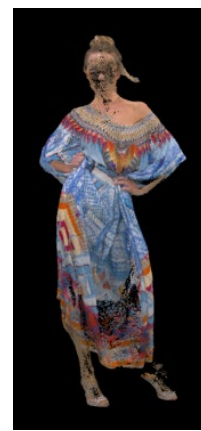

(c)

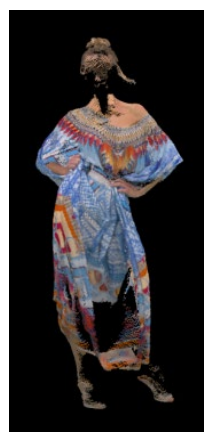
(d)
Figure 2: Longdress_1300 point cloud: (a) original point cloud $(857966=$ $100 \%$ points), (b) decompressed point cloud from equirectangular panorama with $32768 \times 32768$ pixels ( $844980=98.49 \%$ points), (c) decompressed point cloud from equirectangular panorama with $1024 \times 1024$ pixels $(349471=$ $40.73 \%$ points), (d) decompressed point cloud from azimuthal panorama with $1024 \times 1024$ pixels $(305220=35.57 \%$ points $)$

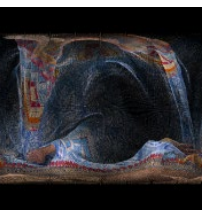

(a)

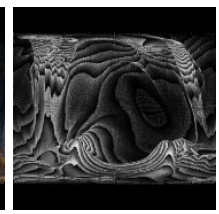

(b)

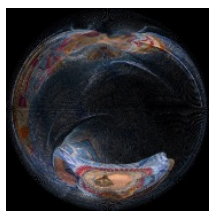

(c)

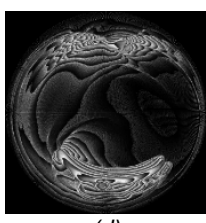

(d)
Figure 3: Longdress_1300 point cloud, panorama images from projection with $1024 \times 1024$ pixels: (a) equirectangular, color panorama, (b) equirectangular, grayscale range, third part, (c) azimuthal, color panorama, (d) azimuthal, grayscale range, third part

\section{Other methods}

Several methods have been proposed where the original PC is first sub-sampled or simplified by selectively discarding points according to some usefulness criteria. An earlier proposal of this type is reported in [27] where the point cloud is first partitioned using a kd-tree. Afterwards, a bounding sphere is computed for each point and a local normal variance of the kd-tree clustered points inside the sphere is computed. Based on this value and some other indicators a decision is taken on whether to remove the point at the centre of the sphere from the point cloud.

Another method that follows a similar approach, i.e. discards less important points, was introduced in [28]. This method resamples an input point cloud based on a measure of the utility of each point for the definition of the implicit surface contours. The algorithm uses a point cloud representation based on graphs on which several features are computed for use in a random resampling of the original point cloud. The features provide a measure of the utility of each point which can then be discarded according to a discard rate and the utility value. Since the remaining points do not change position their attributes information does not need to be further processed.

\section{Background on Objective Measures of Point Cloud Quality}

The quality of a point cloud can be computed based on the geometric distance or distortion between the point could of interest and a reference point cloud. There are several distortion measures described in the literature although only a few are used in the context of coding for compression and all are Full Reference-type quality measures. The most common ones have been described in [29] and are essentially averaged distances between points or local surface approximations in the point cloud under evaluation (henceforth called $\mathrm{PC}_{\mathrm{E}}$ ) and either points or local surfaces in the reference point cloud (called $\mathrm{PC}_{\mathrm{R}}$ from now on). The simplest subset of these measures, the point-to-point measures, are computed by first determining for each point $A$ in $\mathrm{PC}_{\mathrm{E}}$ the closest point in $\mathrm{PC}_{\mathrm{R}}, B$, and measuring their distance, $d(A, B)$. These distances are then combined either by computing their root mean squared average, or by computing the Hausdorff metric over the set of $d(A, B)$ distances. Once these average distances (dRMS or dHausdorff) are computed an objective quality measure can be calculated according to PSNR = $10 \log _{10}\left(\frac{M^{2}}{d_{R M S}}\right)$ for the case of $\mathrm{d}_{\mathrm{RMS}}$ and in a similar fashion for

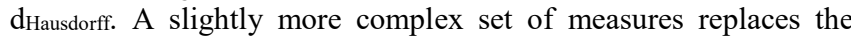
point-to-point distances by point-to-plane distances, where for each point $A$ in $\mathrm{PC}_{\mathrm{E}}$ first the closest point in $\mathrm{PC}_{\mathrm{R}}, B$ is found, after which the distance vector is projected onto the normal vector of the reference point cloud, at point $B$. The length of the projection is the local point-to-plane distance. Once these distances are computed for all points in $\mathrm{PC}_{\mathrm{E}}$ their values are used to compute average distances and PSNR values as described for the point-to-point measures. These latter point-to-plane measures are in general more cumbersome to compute, as they may require local normal vector estimation, an operation that is computationally demanding.

Projection measures involve standard 2D image quality measures such as SSIM, or PSNR being computed on a set of projections (renderings) of the point cloud. Any image quality measure (full, reduced or no reference) can be also applied using the same procedure as described next. Firstly, the point cloud (PC) is rotated and scaled in order to fit in a minimum bounding box of 1 . Afterwards, a surface reconstruction algorithm is used to render a surface fitted to the points of the PC, for example the screened 
Poisson surface reconstruction algorithm [30]. The surface reconstructed $\mathrm{PC}$ can be then imported into a $3 \mathrm{D}$ creation suite that can render projections from PCs, e.g. Blender [31]. Illumination source (lamp) and camera must be placed far enough away from the point cloud so that the surface rendering of the $\mathrm{PC}$ will be uniformly lit over the whole image. For geometry-only PCs, lamp properties must be properly defined, to generate shadings on surface reconstructed object. For geometry and texture PCs, the lamp can be skipped. An arbitrary number of projections can be made using this setup, for both the original and degraded point cloud. Each projection from the original and degraded PC can be then compared using full-reference image quality measures, and the final measure can be calculated as an average measure from all projections. A registration step can be also used, to minimize distance between projections being compared. It can be noted that projections from differently degraded PCs usually will not lie in the same place, because different degradation levels won't produce same surface reconstructed objects, even if they are placed at the origin, prior to projection calculation. A similar proposal can be found in [32]. In this paper, authors developed rendering software, which performs real-time voxelization and projection of the $3 \mathrm{D}$ point clouds, which contain both geometry and texture, onto $2 \mathrm{D}$ planes. Projections are then used by the usual image objective quality metrics, in order to predict the perceptual quality of the displayed stimuli.

\section{Subjective Experiments}

The JPEG committee have performed two rounds of subjective experiments. The first experiment has already been published and will be briefly described here [33] [34]. Results of the second experiment are currently being prepared for publication and will not be described in this paper.

\section{First Subjective Experiment}

The goal of the first subjective experiment was to review the effects of rendering on visual quality of point clouds for a variety of object types for both 2D and 3D visualization. This experiment was divided into two parts:

In part 1 of the experiment, the focus was on the comparison of $3 \mathrm{D}$ objective metrics and subjective judgments for point clouds with and without Poisson Surface Reconstruction [30] rendered using 2D systems [33]. Five laboratories across Europe participated in part 1 of the experiment, in particular, the University of Beira Interior, Portugal (UBI); University of Coimbra, Portugal (UC); University North, Croatia (UNIN); École Polytechnique Fédérale de Lausanne (EPFL), Switzerland and University of Patras (UP), Patras, Greece. The results of this part of the experiment are fully reported in [33].

In part 2 of the experiment, subjective quality judgements for point clouds with and without Poisson Surface Reconstruction [30] were compared between $2 \mathrm{D}$ and $3 \mathrm{D}$ displays. The participants in this part of the experiment were the UBI, UC and UNIN. The results of this part of the experiment are fully reported in [34].

\section{Methodology}

For each part of the experiment, 6 point clouds were selected from JPEG Pleno Point Cloud Database [33] as shown in Figure 4 and compressed by oct-tree pruning at various levels of remaining points $(30 \%, 50 \%, 70 \%, 90 \%)$ achieved by selecting leaf node size [33] [34].

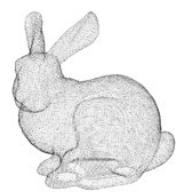

(a)

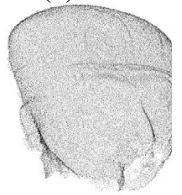

(d)

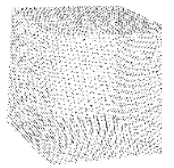

(b)

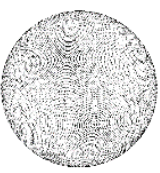

(e)

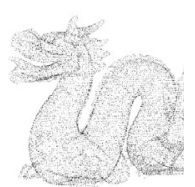

(c)

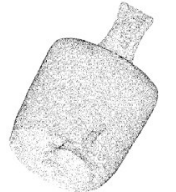

(f)
Figure 4: Point cloud objects used in the first subjective experiment (a) "bunny" from the Stanford 3D repository, (b) "cube" (synthetically generated), (c) "dragon" from the Stanford $3 D$ repository, (d) "Egyptian mask" scanned using an Intel RealSense R200, (e) "sphere" (synthetically generated) and (f) "vase" from the MPEG Inanimate Objects database.

$3 \mathrm{D}$ videos were created for the original and compressed point clouds by rotating the point clouds in predetermined sequences at 1 degree intervals through 720 degrees firstly 360 degrees about the top-bottom axis and then 360 degrees about the left-right axis. The point clouds were then rendered for display to 2D and 3D (part 2 only) [34]. Two forms of rendering were examined. The first form was with the point clouds rendered without surface reconstruction, and the second form was when the point clouds were rendered with Screen Poisson Surface Reconstruction [30].

The original and compressed stimuli videos under the various experimental conditions (with and without surface reconstruction, 2D or 3D display, differing levels of oct-tree pruning) were shown to participants across all the participating laboratories and DMOS scores collected according to ITU-R BT.500-13 [35].

\section{Results}

From this experiment, came a number of key results [33] [34] which will only be summarized briefly here.

Independently of using a 2D or 3D display, similar trends in correlation between subjective scores were observed. The Pearson correlations between subjective scores of different labs are in the ranges of $(0.947,0.989)$ for part 1 and of $(0.944,0.971)$ for part 2 . The Spearman correlations are in the range $(0.838,0.969)$ for part 1 and for part $2(0.843,0.893)$. The correlation results between the $2 \mathrm{D}$ (UBI, UC, UNIN, EPFL and UP) and 3D (UBI, UC and UNIN) subjective evaluations are in the range $(0.941,0.987)$ for Pearson correlation and $(0.804,0.950)$ for Spearman correlations. However, the type of equipment was different for the different tests. The brand, resolution or technology of the display did not affect the results. For instance, in the second experiment UBI used a 3D LG passive display, UC a 3D ASUS active display and UNIN an autostereoscopic Dimenco display.

The objective evaluation did not reveal to represent properly the subjective scores. The best correlation result between the best metric (in this case p2point with Hausdorff distance) and subjective result (UNIN) was 0.834 for Pearson correlation and 0.727 for Spearman correlation obtained without considering the cube results. The cube is an outlier because of its planar structure, that always leads to good reconstructions. Hence, with this rendering method the subjective evaluation did not represent well the perception of quality given by the subjective tests.

Finally, the subjective results were also compared with a previous study [35] where no surface reconstruction was made. The results obtained in [32] revealed a poor correlation ( 0.8 for Pearson 
and 0.73 for Spearman), inducing that the rendering method has a large influence on the quality perception. New studies are required to understand the best rendering method for reliable quality studies.

Readers are referred to [33] and [34] for detailed analysis of the results of these experiments.

\section{Conclusions and Future Work}

The JPEG Pleno Point Cloud Ad hoc Group have made progress in surveying the state of the art in coding and quality evaluation for point clouds and identifying key use-cases and key requirements. Initial subjective experiments have been performed that will help to guide the evaluation of future proposals. However, there is still much work to be done to determine the best protocols for subjective testing and it is clear that subjective judgments can be strongly affected by content as well as whether surface reconstruction is used prior to rendering.

In regard to point cloud quality measures, projection-based image quality measures show particular promise, but there is still work to do on finding the best measure and mitigating the effects of surface reconstruction and lighting direction on predicting subjective judgments using objective metrics.

Work in JPEG continues, particularly in the following areas:

- Identifying use-cases and requirements that would allow a Call for Proposals to be created.

- Determining the best subjective testing protocol and objective metrics to allow for fair and relevant testing of proposals.

- Developing a Common Test Conditions Document to guide evaluation of future point cloud coding proposals in a fair and consistent manner.

JPEG is actively seeking advice and support from industry and academia for all of the above to help make future standards in this area as relevant as possible.

\section{Acknowledgments}

The authors would like to acknowledge the assistance of $8 \mathrm{i}$ Labs, Microsoft, Universidad Politécnica de Madrid, ScanLAB Projects, and the University of São Paulo for supplying point clouds for the JPEG Pleno Point Cloud Database. We would also like to acknowledge the authors of [33] and [34], and in particular Prof Touradj Ebrahimi, JPEG Convenor, and Evangelos Alexiou for permission to describe the details of the subjective testing conducted for ISO/IEC/SC29/WG1.

\section{References}

[1] ISO/IEC/SC29/WG1 (JPEG) Committee, "JPEG Pleno Database," 2019. [Online]. Available: https://jpeg.org/plenodb/. [Accessed 62 2019].

[2] J. Peng and C.-C. J. Kuo, "Geometry-guided progressive lossless 3d mesh coding with octree (ot) decomposition," ACM Transactions on Graphics, vol. 24, no. 3, pp. 609-616, July 2005.

[3] A. Hornung, K. M. Wurm, M. Bennewitz, C. Stachniss and W. Burgard, "Octomap: an efficient probabilistic 3d mapping framework based on octrees," Autonomous Robots, vol. 34, no. 3, p. 189-206, April 2013.
[4] R. B. Rusu and S. Cousins, "3D is here: Point Cloud Library (PCL)," in 2011 IEEE International Conference on Robotics and Automation, Shanghai, China, 2011.

[5] R. Schnabel and R. Klein, "Octree-based point-cloud compression," in SPBG'06 Proceedings of the 3rd Eurographics / IEEE VGTC conference on Point-Based Graphics, Boston, Massachusetts, USA, 2006.

[6] Y. Huang, J. Peng, C.-C. J. Kuo and M. Gopi, "A Generic Scheme for Progressive Point Cloud Coding," IEEE Transactions on Visualization and Computer Graphics, vol. 14, no. 2, pp. 440 - 453, 2008.

[7] W. Jiang, J. Tian, K. Cai, F. Zhang and T. Luo, "Tangent-planecontinuity maximization based 3D point compression," in 201219 th IEEE International Conference on Image Processing, Orlando, FL, USA, 2012.

[8] J. Smith, G. Petrova and S. Schaefer, "SMI 2012: Full Progressive encoding and compression of surfaces generated from point cloud data," Computers and Graphics, vol. 36, no. 5, pp. 341-348, 2012.

[9] J. Kammerl, N. Blodow , R. B. Rusu, S. Gedikli, M. Beetz and E. Steinbach, "Real-time compression of point cloud streams," in 2012 IEEE International Conference on Robotics and Automation, Saint Paul, MN, USA, 2012.

[10] R. L. de Queiroz and P. A. Chou, "Compression of 3D Point Clouds Using a Region-Adaptive Hierarchical Transform," IEEE Transactions on Image Processing , vol. 25, no. 8, pp. 3947 - 3956, 2016.

[11] J. Peng, C. Kim and C.-C. J. Kuo, "Technologies for 3d mesh compression: A survey," Journal of Visual Communication and Image Representation, vol. 16, no. 6, p. 2005, 688-733.

[12] P. Alliez and C. Gotsman, "Recent Advances in Compression of 3D Meshes," in Advances in Multiresolution for Geometric Modelling, Berlin, Germany, Springer, Berlin, Heidelberg, 2005, pp. 3-26.

[13] A. Maglo, G. Lavoué, F. Dupont and C. Hudelot, "3D Mesh Compression: Survey, Comparisons, and Emerging Trends," ACM Computing Surveys (CSUR), vol. 47, no. 3, pp. 44:1-44:41, 2015.

[14] S. Rusinkiewicz and M. Levoy, "QSplat: a multiresolution point rendering system for large meshes," in SIGGRAPH' 00 Proceedings of the 27th annual conference on Computer graphics and interactive techniques, New Orleans, Louisiana, USA, 2000.

[15] J. Rossignac, "Edgebreaker: connectivity compression for triangle meshes," IEEE Transactions on Visualisation and Computer Graphics, vol. 5, no. 1, pp. 47-61, 1999.

[16] Z. Karni and C. Gotsman, "Spectral compression of mesh geometry," in SIGGRAPH '00 Proceedings of the 27th annual conference on Computer graphics and interactive techniques, New Orleans, Louisiana, USA, 2000.

[17] C. Zhang, D. Florêncio and C. Loop, "Point cloud attribute compression with graph transform," in 2014 IEEE International Conference on Image Processing (ICIP), Paris, France, 2014.

[18] R. A. Cohen, D. Tian and A. Vetro, "Point cloud attribute compression using 3-d intra prediction and shape-adaptive 
transforms," in 2016 Data Compression Conference (DCC), Snowbird, UT, USA, 2016.

[19] D. Thanou, P. A. Chou and P. Frossard, "Graph-Based Compression of Dynamic 3D Point Cloud Sequences," IEEE Transactions on Image Processing, vol. 25, no. 4, pp. 1765-1778, 2016.

[20] R. L. de Queiroz and P. A. Chou, "Transform Coding for Point Clouds Using a Gaussian Process Model," IEEE Transactions on Image Processing, vol. 26, no. 7, pp. 3507-3517, 2017.

[21] P. d. O. Rente, C. Brites, J. M. Ascenso and F. M. B. Pereira, "Graph-Based Static 3D Point Clouds Geometry Coding," IEEE Transactions on Multimedia, vol. 21, no. 2, pp. 284 - 299, 2018.

[22] L. He, W. Zhu and Y. Xu, "Best-effort projection based attribute compression for 3D point cloud," in 2017 23rd Asia-Pacific Conference on Communications (APCC), Perth, WA, Australia, 2017.

[23] H. Houshiar, D. Borrman, J. Elseberg and A. Nüchter, "Panorama based point cloud reduction and registration," in 2013 16th International Conference on Advanced Robotics (ICAR), Montevideo, Uruguay, 2013.

[24] H. Houshiar and A. Nüchter, "3D point cloud compression using conventional image compression for efficient data transmission," in 2015 XXV International Conference on Information, Communication and Automation Technologies (ICAT), Sarajevo, Bosnia and Herzegovina, 2015.

[25] E. d'Eon, B. Harrison, T. Myers and P. A. Chou, "8i Voxelized Full Bodies - A Voxelized Point Cloud Dataset," ISO/IEC JTC1/SC29 Joint WG11/WG1 (MPEG/JPEG) input document WG11M40059/WG1M74006, Geneva, Switzerland, 2017.

[26] A. Nüchter, "3DTK - The 3D Toolkit," Universität Würzburg, 2019. [Online]. Available: http://slam6d.sourceforge.net/. [Accessed 5 February 2019].

[27] Z. Xiao and W. Huang, "Kd-tree Based Nonuniform Simplification of 3D Point Cloud," in 2009 Third International Conference on Genetic and Evolutionary Computing, Guilin, China, 2009.

[28] S. Chen, D. Tian, C. Feng, A. Vetro and J. Kovačević, "Fast Resampling of Three-Dimensional Point Clouds via Graphs," IEEE Transactions on Signal Processing, vol. 66, no. 3, pp. 666 - 681, 2018.

[29] E. Dumic, C. R. Duarte and d. S. C. A. Luis, "Subjective evaluation and objective measures for point clouds - State of the art," in First International Colloquium on Smart Grid Metrology (SmaGriMet), Split, Croatia, 2018.

[30] M. Kazhdan and H. Hoppe, "Screen poisson surface reconstruction," ACM Transactions on Graphics, vol. 32, no. 3, pp. 29:1-29:13, 2013.

[31] Blender Foundation, "blender.org - Home of the Blender Project Free and Open 3D Creation Software," 2019. [Online]. Available: https://www.blender.org/. [Accessed 7 February 2019].

[32] E. M. Torlig, E. Alexiou, T. A. Fonseca, R. L. de Queiroz and T. Ebrahimi, "A novel methodology for quality assessment of voxelized point clouds," in SPIE vol. 10752, Applications of Digital Image Processing XLI, San Diego, California, USA, 2018.

[33] E. Alexiou, M. V. Bernardo, L. A. d. S. Cruz, C. Duarte, E. Dumic, T. Ebrahimi, D. Matkovic, M. Pereira, A. Pinheiro and A. Skodras, "Point Cloud Subjective Evaluation Methodology based on 2D Rendering," in 2018 Tenth International Conference on Quality of Multimedia Experience (QoMEX), Cagliari, Italy, 2018.

[34] E. Alexiou, A. M. G. Pinheiro, C. Duarte, D. Matkovic, E. Dumic, L. A. d. S. Cruz, L. G. Dmitrovic, M. V. Bernado, M. Pereira and T. Ebrahimi, "Point cloud subjective evaluation methodology based on reconstructed surfaces," in SPIE Optical Engineering + Applications 2018, SPIE vol. 10752, San Diego, California, United States, 2018.

[35] ITU-R, "Recommendation ITU-R BT.500-13 Methodology for the subjective assessment of the quality of television pictures," International Telecommunication Union, Geneva, Switzerland, 2012.

\section{Author Biographies}

Stuart Perry received his BEng(Hons) (1994) in electrical engineering and his PhD (1999) both from University of Sydney. Since then he has worked in the Maritime Operations Division of the Defence Science and Technology Organisation and Canon Information Systems Research Australia (CiSRA). He currently is an Associate Professor in the School of Electrical and Data Engineering, Faculty of Engineering and IT, University of Technology Sydney. His work has focused on image quality and human perception. He is a member of IS\&T, SPIE and IEEE.

Antonio Pinheiro is an Assistant Professor at U.B.I. (Universidade da Beira Interior), Covilhã, Portugal and a researcher at I.T. (Instituto de Telecomunicações), Portugal. He received the BSc (Licenciatura) from I.S.T., Lisbon in 1988 and the PhD in Electronic Systems Engineering from University of Essex in 2002. Currently, his research interests are on Image Processing, namely on Multimedia Quality Evaluation, Emerging $3 D$ Imaging Technologies, and Medical Image Analysis. He is a Portuguese delegate to ISO/IEC JTC1/SC29, involved with JPEG standardisation committee, where he is currently the Communication Subgroup chair and participates in JPEG Pleno development. He is a senior member of IEEE.

Emil Dumic is an Assistant Professor at the University North, Department of Electrical Engineering. He received his $P h D$ degree from University of Zagreb, Faculty of Electrical Engineering and Computing in 2011. His research interests include development of objective image and video quality measures, image, video and $3 D$ video quality assessment databases, image interpolation, DVB, point clouds etc.

Luis A. da Silva Cruz, received the Licenciado and M.Sc. degrees in Electrical Engineering from the University of Coimbra, Portugal, in 1989 and 1993 respectively. He also holds an MSc degree in Mathematics and a Ph.D. degree in Electrical Computer and Systems Engineering from Rensselaer Polytechnic Institute (RPI), Troy, NY, US granted in 1997 and 2000 respectively. He has been with the Department of Electrical and Computer Engineering of the University of Coimbra in Portugal as an Assistant Professor since 2000. He is a researcher of the Institute for Telecommunications of Coimbra where he works on video processing and coding and medical image processing. He is a member of the EURASIP, SPIE and IEEE technical societies. 